\title{
TECNOLOGIAS EDUCACIONAIS NO ENSINO DA ENFERMAGEM DURANTE A PANDEMIA POR COVID-19: REVISÃO SISTEMÁTICA
}

\section{EDUCATIONAL TECHNOLOGIES IN TEACHING NURSING DURING THE PANDEMIC BY COVID-19: A SYSTEMATIC REVIEW}

\section{Raphaella Castro Jansen ${ }^{1} *$ Vitória Costa Oliveira ${ }^{2} *$ Maria Rayssa do Nascimento Nogueira $^{3}$ Iorana Candido da Silva ${ }^{*}$ José Erivelton de Souza Maciel Ferreira ${ }^{*}$ Tahissa Frota Cavalcante ${ }^{6} *$ Ana Luisa Brandão de Carvalho Lira ${ }^{7}$}

\section{RESUMO}

Objetivo: Analisar as tecnologias educacionais utilizadas no ensino de Enfermagem durante a pandemia por COVID-19. Métodos: Revisão sistemática de métodos mistos realizada no Portal PubMed, Portal Regional da Biblioteca Virtual em Saúde, no buscador acadêmico Science Direct, no banco de dados da Cochrane e nas bases de dados CINAHL, Scopus e Web of Science. Utilizaram-se os descritores controlados em saúde: Tecnologia Educacional, Enfermagem, COVID-19 e seus sinônimos em inglês. Resultados: A busca resultou na captura de 40 artigos, dos quais quatro compuseram a amostra final por atenderem aos critérios de inclusão e exclusão estabelecidos. Constatou-se que as tecnologias educacionais mais utilizadas foram encontros virtuais, podcasts, gamificação individual ou em grupo, exames eletrônicos remotos, simulações realísticas e casos clínicos. Conclusões: As tecnologias educativas utilizadas no ensino de Enfermagem durante a pandemia por COVID-19 são alternativas eficazes para a continuidade da formação, por possibilitarem ampliação da qualidade do ensino, desenvolvimento do raciocínio diagnóstico, estímulo ao diálogo e criatividade dos discentes e docentes de enfermagem nesse contexto emergencial. Entretanto, apesar das possibilidades proporcionadas, a experiência prática nos serviços de saúde é indispensável.

Palavras-chave: COVID-19; Educação em Enfermagem; Enfermagem; Ensino; Pandemia; Tecnologia educacional.

\footnotetext{
ABSTRACT

Objective: To analyze the educational technologies used in teaching Nursing during the pandemic by COVID-19. Method: Systematic review of mixed methods performed in the Pubmed Portal, Regional Portal of the Virtual Health Library, in the academic search Science Direct, in the Cochrane database and in the CINAHL, Scopus and Web of Science databases. Health-controlled descriptors were used: Educational Technology, Nursing, COVID-19 and its synonyms in English. Results: The search resulted in the capture of 40 articles, of which four comprised the final sample for meeting the inclusion and exclusion criteria established. It was found that the most used educational technologies were virtual meetings, podcasts, individual or group gamification, remote electronic tests, realistic simulations and clinical cases. Conclusions: The educational technologies used in teaching Nursing during the pandemic by COVID-19 are effective alternatives for the continuity of training, as they make possible to increase the quality of teaching, development of diagnostic reasoning, encouraging the dialogue and creativity of students and nursing teachers in this emergency context. However, despite the possibilities provided, practical experience in health services is indispensable.

Keywords: COVID-19; Education, Nursing; Educational Technology; Pandemic; Nursing; Teaching.

${ }^{1}$ Universidade da Integração Internacional da Lusofonia Afro-Brasileira, Redenção, Brasil. ORCID: https://orcid.org/0000-00024032-5825

${ }^{2}$ Universidade da Integração Internacional da Lusofonia Afro-Brasileira, Redenção, Brasil. ORCID: https://orcid.org/0000-00017341-8596

${ }^{3}$ Universidade da Integração Internacional da Lusofonia Afro-Brasileira, Redenção, Brasil. ORCID: https://orcid.org/0000-00030355-5901

${ }^{4}$ Universidade da Integração Internacional da Lusofonia Afro-Brasileira, Redenção, Brasil. ORCID: https://orcid.org/0000-00030815-1703

${ }^{5}$ Universidade da Integração Internacional da Lusofonia Afro-Brasileira, Redenção, Brasil. ORCID: https://orcid.org/0000-00032668-7587

${ }^{6}$ Universidade da Integração Internacional da Lusofonia Afro-Brasileira, Redenção, Brasil. ORCID: https://orcid.org/0000-00022594-2323

${ }^{7}$ Universidade Federal do Rio Grande do Norte, Natal, Brasil. ORCID: https://orcid.org/0000-0002-7255-960X
} 


\section{INTRODUÇÃO}

Em virtude das inúmeras consequências sociais, econômicas e políticas que vêm acarretando à sociedade, a pandemia por COVID-19 configura-se como um dos mais importantes problemas de saúde pública da atualidade. No Brasil, esse cenário impactou de forma drástica $\mathrm{o}$ processo educacional dos cursos da saúde, tendo em vista que as aulas presenciais foram suspensas exigindo uma reestruturação no método de ensino-aprendizagem ${ }^{(1)}$.

Diante desse cenário, o Brasil instituiu a portaria $\mathrm{n}^{\circ} 345 / 2020$ do Ministério da Educação, permitindo a continuidade do ensino por meio de aulas remotas e com a aplicação de Tecnologias de Informação e Comunicação $\quad(\text { TIC })^{(2)}$. As TIC são ferramentas e recursos ligados à cibercultura, que possibilitam o compartilhamento e o armazenamento de informações de meios de comunicação de massa $^{(3)}$. Dessa forma, sua utilização configura-se como uma adaptação emergencial, em que o ambiente educacional foi drasticamente modificado e o ensino remoto foi iniciado.

No âmbito da educação em enfermagem, o dilema torna-se maior devido à urgente necessidade de formar novos profissionais em virtude da carência global por enfermeiros ${ }^{(4)}$. Ademais, a formação do enfermeiro na graduação é de suma importância para a saúde pública e, até mesmo, para o setor econômico, tendo em vista que esse profissional responde por metade da força de trabalho global ${ }^{(5)}$.

Nesse contexto, as Instituições de Ensino Superior (IES) e os docentes tiveram que remodelar a metodologia de ensino por meio do uso de tecnologias educacionais em ambientes virtuais e meios digitais ${ }^{(6)}$. As tecnologias educacionais são artefatos presentes dentro das escolas que favorecem o compartilhamento e a obtenção de conhecimento $^{(7)}$.

Desse modo, as práticas pedagógicas e táticas de ensino migraram para o ambiente on-line, havendo a necessidade de adaptação de professores e alunos a essa nova realidade de ensino ${ }^{(8)}$. Esta modificação acentuou problemas crônicos na educação brasileira, tais como: desigualdade de acesso a recursos tecnológicos por parte dos discentes, questões socioeconômicas que interferem na qualidade do estudo, forma de avaliação e despreparo dos profissionais da educação para o manuseio das $\mathrm{TIC}^{(2,9-10)}$.

Frente ao novo contexto imposto pela necessidade de distanciamento social, essa problemática se intensifica no ensino da enfermagem, por ser uma profissão que requer a inserção do estudante nos cenários de prática do sistema único de saúde desde o início da formação. Além disso, sua formação exige a integração do tripé ensino, serviço e comunidade, uma vez que a relação entre 
estes também permite uma inserção precoce do aluno no campo de prática. A vista disso é indiscutível a necessidade de proporcionar uma forma de ensino que potencialize a autonomia, a participação e capacidade crítico-reflexiva desses futuros profissionais, o que aumenta o desafio no ensino à distância ${ }^{(5,11)}$.

Assim, considerando a importância de ofertar um ensino de qualidade que supra as necessidades acadêmicas mesmo diante da crise sanitária, este estudo mostra-se relevante por verificar o que vem sendo produzido e discutido acerca da temática, podendo mapear a implementação das tecnologias no ensino e, desse modo, contribuir com a melhor utilização de recursos e o fortalecimento da formação em enfermagem.

Ante o exposto, esta revisão tem por objetivo analisar as tecnologias educacionais utilizadas no ensino de Enfermagem durante a pandemia por COVID-19.

\section{MÉTODOS}

Trata-se de uma revisão sistemática de métodos mistos, que permite a seleção, avaliação e síntese de conhecimentos de estudos com diferentes delineamentos. Esse método facilita o acesso a informações para a tomada de decisões a partir de uma prática baseada em evidências seguindo critérios metodológicos bem definidos ${ }^{(12-13)}$.
Desse modo, o presente estudo foi realizado a partir das seguintes etapas: 1) formulação da pergunta norteadora; 2) definir dos critérios de inclusão e exclusão; 3) aplicar estratégia de busca; 4) selecionar artigos por meio de avaliação de título e resumo; 5) selecionar artigos com base no texto completo; 6) avaliar a qualidade dos estudos incluídos; 7) Sintetizar amostra selecionada ${ }^{(12)}$.

A formulação da questão de pesquisa foi inspirada na estratégia PICo (População, Interesse, Contexto) ${ }^{(14)}$, definindo como População - ensino de Enfermagem; Interesse - tecnologias educacionais; Contexto pandemia por COVID-19. A questão norteadora adotada para identificação do problema foi: "Quais tecnologias educacionais estão sendo utilizadas no ensino de Enfermagem durante a pandemia por COVID-19?”.

A busca na literatura foi realizada por dois pesquisadores de forma concomitante no período de abril de 2021, através do portal U.S. National Library of Medicine (PubMed); Portal Regional da Biblioteca Virtual em Saúde (BVS); no buscador acadêmico Science Direct; no banco de dados da Cochrane e das bases de dados Cumulative Index to Nursing and Allied Health Literature (CINAHL), Scopus e Web of Science (WOS). O acesso às fontes de pesquisas ocorreu por acesso remoto via Comunidade Acadêmica Federada 
(CAFe), através do portal de periódicos da Coordenação de Aperfeiçoamento de Pessoal de Nível Superior (CAPES), do Ministério da Educação (MEC).

Para estratégia de busca, utilizaram-se descritores controlados concatenados com o operador booleano $A N D$, dispostos no Medical Subject Headings (MeSH) e no Descritores das Ciências da Saúde (DeCS), sem determinar um campo específico de busca, optando por all fields. As combinações utilizadas estão descritas no quadro 1 .

Quadro 1- Estratégias de buscas utilizadas na Science Direct, PubMed, Web of Science, Scopus, CINAHL, Cochrane e Biblioteca Virtual da Saúde. Redenção, CE, Brasil, 2021.

\begin{tabular}{|l|l|}
\hline \multicolumn{1}{|c|}{ FONTE } & \multicolumn{1}{|c|}{ ESTRATÉGIAS DE BUSCA } \\
\hline $\begin{array}{l}\text { Science Direct; PubMed; W } \\
\text { of Science; Scopus; CINAHL; } \\
\text { Cochrane. }\end{array}$ & $\begin{array}{l}\text { Educational Technology AND Nursing AND } \\
\text { COVID-19 }\end{array}$ \\
\hline Biblioteca Virtual da Saúde & $\begin{array}{l}\text { Tecnologia Educacional AND Enfermagem AND } \\
\text { COVID-19 }\end{array}$ \\
\end{tabular}

Fonte: Dados da pesquisa.

Consideraram-se como critérios de inclusão estudos disponíveis eletronicamente na íntegra, em recorte atemporal e em qualquer idioma que fossem relacionados ao objeto de pesquisa. Foram excluídos artigos oriundos de revisões, editoriais, teses $\mathrm{e}$ dissertações. Os artigos que se repetiram entre as bases foram considerados apenas uma vez.

Os artigos resultantes da aplicação da estratégia de busca foram exportados das fontes de pesquisa para o Rayyan, software que auxilia no arquivamento, organização e seleção de referências bibliográficas ${ }^{(15)}$. A seleção primária dos estudos ocorreu por meio da leitura minuciosa de títulos e resumos, sendo incluídos os que atendiam aos critérios de inclusão estabelecidos. Posteriormente, realizou-se a leitura dos artigos na íntegra para compor a amostra final. Todo o processo de busca e seleção foi realizado por duas pesquisadoras de forma independente. Em caso de dúvida ou discrepância entre elas, um terceiro pesquisador foi consultado. A seleção dos estudos seguiu as recomendações do checklist do Statement for Reporting Systematic Review and Meta-Analyses of Studies - PRISMA ${ }^{(16)}$.

Para a avaliação da qualidade metodológica utilizou-se o instrumento Mixed Methods Appraisal Tool (MMAT), versão 2011, que permite a análise simultânea de estudos com diversos tipos de delineamento. 
O MMAT é composto por 20 critérios de qualidade divididos nos cinco tipos de estudos: qualitativo, quantitativo clínico aleatorizado (ensaios), quantitativo nãoaleatório, quantitativo descritivo e estudos mistos ${ }^{(12)}$. A pontuação final foi obtida conforme o delineamento do estudo, considerando $25 \%$ para cada um dos quatro critérios avaliados, de acordo com cada tipo metodológico.

Com relação à coleta e análise dos dados, utilizou-se de um formulário elaborado pelos próprios autores, organizado por meio de um quadro sinóptico com as seguintes variáveis: título do artigo, autores, ano e país de publicação, delineamento do estudo, nível de Evidência, avaliação da qualidade metodológica e principais resultados.

A avaliação do nível de Evidência dos estudos foi realizada de acordo com à categorização proposta por Melnyk e Fineout-
Overholt (2005) $)^{(17)}$, que classifica os estudos em seis níveis, a saber: I - metanálise de estudos controlados e randomizados; nível II - estudo experimental; nível III - estudo quase experimental; nível IV - estudo descritivo/não experimental ou com abordagem qualitativa; nível $\mathrm{V}$ - relato de caso ou experiência; nível VI - consenso e opinião de especialistas.

\section{RESULTADOS}

$\mathrm{O}$ processo de amostragem para a identificação dos estudos da pesquisa está representado na Figura 1, por meio de um fluxograma, que expõe a identificação e seleção dos artigos em cada base de dados. A estratégia de busca aplicada possibilitou a captura de 40 artigos, dos quais quatro compuseram a amostra final por atenderem aos critérios de inclusão e exclusão estabelecidos. 
Figura 1- Fluxograma de identificação e seleção das publicações de acordo com o PRISMA 2020. Redenção, CE, Brasil, 2021.

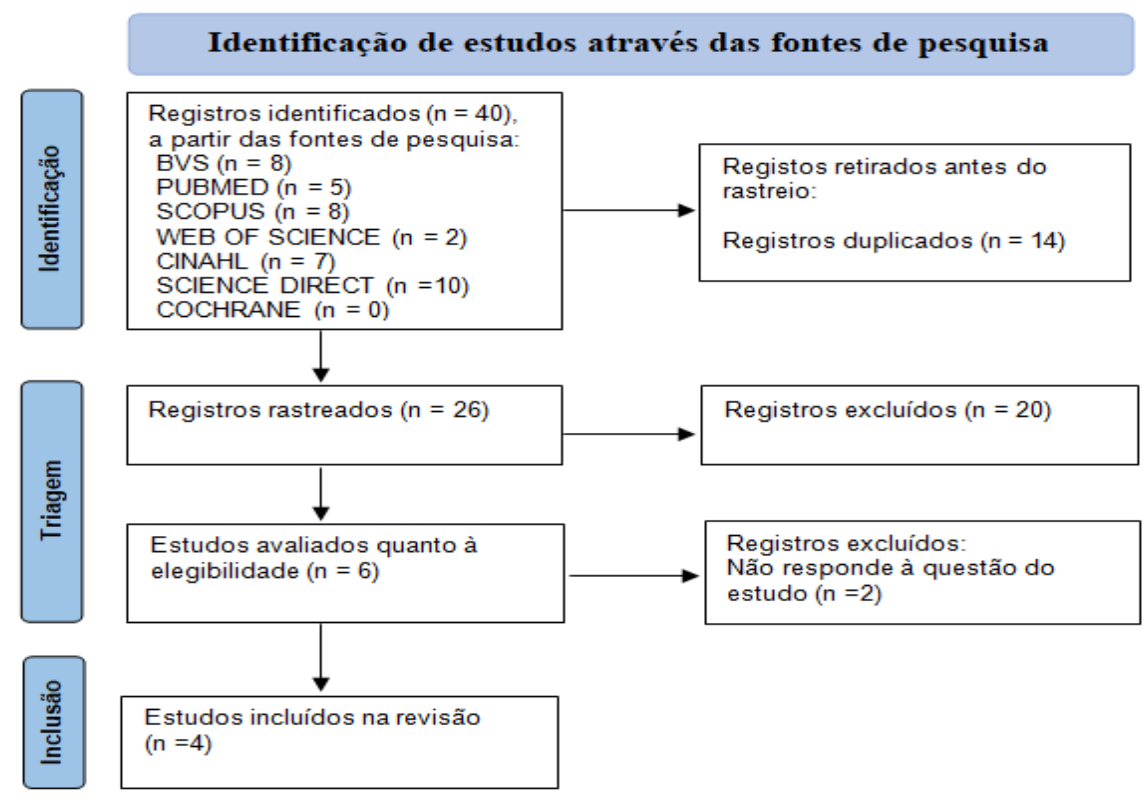

Fonte: Modelo adaptado do fluxograma PRISMA, 2020.

Desses quatro estudos, três $(75 \%)$ apresentavam-se na língua inglesa e apenas um $(25 \%)$ em língua portuguesa. Referente à procedência, cada estudo foi publicado em um país, sendo um no Brasil (25\%), um no Reino Unido (25\%), um na Jordânia (25\%) e um (25\%) nos Estados Unidos. Quanto à fonte de pesquisa, dois estudos (50\%) foram publicados em periódicos indexados na
CINAHL, um (25\%) na Science Direct e um (25\%) na BVS. No que tange ao nível de evidência, três $(75 \%)$ apresentaram nível $\mathrm{V}$ relacionado a evidências derivadas de relato de experiência e um (25\%) nível IV caracterizado por ser um estudo descritivo/não experimental. A tabela 1 apresenta a síntese dos artigos selecionados para o estudo.

Tabela 1- Distribuição dos artigos incluídos com relação a autores, ano, título, desenho do estudo, nível de evidência e qualidade metodológica. Redenção, CE, Brasil, 2021.

$\begin{array}{ccccc} & & \text { Abordagem } & \\ \text { Estudo } & \text { metodológica/ } & \text { Avaliação } \\ & \text { Ano } & \text { Título } & \text { Desenho do } & \text { MMAT } \\ & & \text { estudo/ Nível de } & \\ & & \text { Evidência } & \end{array}$




\begin{tabular}{|c|c|c|c|c|}
\hline E1 & $\begin{array}{c}\text { Silveira et al. } \\
2020^{18}\end{array}$ & $\begin{array}{lll}\text { Estratégias e desafios do } \\
\text { ensino } & \text { remoto } \\
\text { Enfermagem } & \end{array}$ & $\begin{array}{l}\text { Qualitativo } \\
\text { Relato de } \\
\text { experiência } \\
\text { Nível V. }\end{array}$ & $100 \%$ \\
\hline E2 & $\begin{array}{c}\text { Leigh } \text { et al. } \\
2020^{19}\end{array}$ & $\begin{array}{l}\text { Redefining undergraduate } \\
\text { nurse teaching during the } \\
\text { coronavirus pandemic: use of } \\
\text { digital Technologies }\end{array}$ & $\begin{array}{l}\text { Qualitativo } \\
\text { Relato de } \\
\text { experiência } \\
\text { Nível V. }\end{array}$ & $50 \%$ \\
\hline E3 & $\begin{array}{l}\text { Elsalem et al. } \\
\qquad 2021^{20}\end{array}$ & $\begin{array}{l}\text { Remote E-exams during } \\
\text { Covid-19 pandemic: A cross- } \\
\text { sectional study of students' } \\
\text { preferences and academic } \\
\text { dishonesty in faculties of } \\
\text { medical sciences }\end{array}$ & $\begin{array}{l}\text { Quantitativo não- } \\
\text { aleatório } \\
\text { Estudo transversal. } \\
\text { Nível IV. }\end{array}$ & $100 \%$ \\
\hline E4 & $\begin{array}{l}\text { Konrad; } \\
\text { Fitzgerald; } \\
\text { Deckers. } \\
2021^{21}\end{array}$ & $\begin{array}{l}\text { Nursing fundamentals - } \\
\text { supporting clinical } \\
\text { competency on-line during the } \\
\text { COVID-19 pandemic }\end{array}$ & $\begin{array}{l}\text { Qualitativo } \\
\text { Relato de } \\
\text { experiência } \\
\text { Nível V. }\end{array}$ & $100 \%$ \\
\hline
\end{tabular}

Fonte: Dados da pesquisa.

Dos quatro estudos incluídos, três (75\%) são qualitativos e um (25\%) é quantitativo não-aleatório. Os estudos $\mathrm{E}^{(18)}$, $E 3^{(20)}$ e $E 4^{(21)}$ receberam uma pontuação de $100 \%$ sobre a escala de avaliação da qualidade metodológica MMAT, demonstrando alta qualidade metodológica, uma vez que, todos os quatro critérios foram cumpridos. O estudo E2 ${ }^{19}$ obteve pontuação de $50 \%$, caracterizada pela perda de pontos quanto a não representatividade da população estudada e por não apresentar uma consideração apropriada na análise e interpretação dos resultados.

Diversas foram as tecnologias utilizadas durante $\mathrm{o}$ ensino remoto em enfermagem. No que diz respeito a esse aspecto, tem-se que as atividades mais realizadas a partir das tecnologias digitais foram encontros virtuais, como videoconferências, webnars, lives $\mathrm{e}$ reuniões ${ }^{(18-19)}$, que puderam ser realizadas por 
meio das plataformas Zoom®, Microsoft Teams ${ }^{\circledR}$, e YouTube ${ }^{\circledR}{ }^{(19,21)}$; podcasts através do SoundCloud®, gamificação individual ou coletiva por meio do Kahoot! e Mentimeter ${ }^{\circledR(19)}$; $\quad$ exames eletrônicos remotos $^{(20)}$; além de simulações realísticas virtuais que objetivavam o aprimoramento do raciocínio clínico a partir da elaboração de um plano de cuidados por meio da discussão de casos clínicos por vídeo e utilização do MedEdu À Easy Auscultation para treino dos sons de Korotkoff ${ }^{(21)}$.

Os estudos $\mathrm{E} 1^{(18)}$ e $\mathrm{E} 2^{(19)}$ evidenciaram que as estratégias utilizadas a partir da utilização de tecnologias educacionais interativas viabilizaram o ensino, podendo ser utilizadas para cumprir componente teórico do programa de graduação em enfermagem com qualidade. Além disso, tornaram o processo prazeroso e acessível, uma vez que mostraram-se como facilitadoras para o ensino remoto. $\mathrm{O}$ estudo $\mathrm{E} 3^{(20)}$ utilizou a combinação de exames e questionários e constatou que esse foi o método de avaliação preferido pelos alunos durante o ensino remoto. O estudo $\mathrm{E}^{(21)}$ concluiu que as habilidades de raciocínio clínico dos discentes foram melhoradas a partir da experiência da utilização de tecnologias educativas.

\section{DISCUSSÃO}

Os principais resultados dos estudos analisados mostraram que no atual cenário de pandemia COVID-19, no qual as aulas presenciais foram suspensas, desenvolver estratégias para a continuidade do ensino tornou-se um desafio para o sistema educacional. Em decorrência da impossibilidade de realização de aulas presenciais, as instituições de ensino necessitaram adotar o ensino remoto de forma emergencial visando a redução dos impactos que a suspensão das aulas presenciais causou na rotina dos alunos ${ }^{(5)}$.

Um ensaio crítico sobre uma nota técnica da Organização da Sociedade Civil Todos Pela Educação de $2020^{(22)}$ discutiu as diferenças de conceito entre os termos ensino remoto e Ensino à Distância $(\mathrm{EaD})$. O estudo aponta que o primeiro refere-se a uma forma de ensino que foi adotada em virtude da crise atual e o segundo é uma modalidade de ensino estruturada, com organização de currículo, avaliações e materiais de apoio.

Segundo Silva e colaboradores $(2021)^{(5)}$, o EaD para a Enfermagem é um tema conflituoso, pois embora possua uma estrutura organizacional bem definida ${ }^{(22)}$, essa modalidade de ensino traz prejuízos à qualidade do ensino. Isso ocorre devido à deficiência de capacitação prática, reduzida interação entre aluno e professor e por não favorecer a integração do ensino entre os alunos e os serviços de saúde. Os autores ainda alertam sobre o posicionamento contrário do Conselho Nacional de Saúde, da 
Associação Brasileira de Enfermagem e do

Conselho Federal de Enfermagem à autorização do $\mathrm{EaD}$, até antes da pandemia, frente aos riscos à sociedade decorrentes dos déficits na qualidade do ensino ${ }^{(5)}$.

$\mathrm{O}$ desacordo estrutura-se a partir do princípio de que o enfermeiro deve estar capacitado para prestar uma assistência técnico-científica, sobretudo humanística, devendo conhecer as necessidades de saúde das pessoas, sendo isso dificultado no ensino puramente digital. A integração de conhecimento teórico-prático com a realidade da diversidade dos cenários sociais é prejudicada quando a formação não é presencial. Diante disso, é necessária cautela na adoção da modalidade $\mathrm{EaD}$, considerando as fragilidades que podem ocorrer principalmente no estabelecimento de vínculos entre profissional e paciente, uma vez que inerentemente a Enfermagem associa o conhecimento científico a sensibilidade com as necessidades humanas ${ }^{(10)}$.

Entretanto, por efeito das medidas de prevenção da COVID-19 e da necessidade de continuação da formação acadêmica, as IES adotaram a modalidade remota de forma emergencial. Todas as IES experienciaram essa dificuldade na implantação do ensino remoto, mesmo aquelas com suporte, por exigir do docente e discente a adaptação à nova modalidade, o que torna o processo exaustivo $^{(23)}$. Os docentes precisaram aperfeiçoar sua capacidade de inovação, sistematização do conhecimento e avaliação do processo de aprendizagem dos alunos a partir da inclusão de ferramentas com potencial para melhoria da qualidade de ensino $^{(24)}$.

Ainda que já utilizadas anteriormente, o uso de tecnologias educacionais on-line vem sendo amplamente discutido ${ }^{(25)}$, isso porque o rendimento e aproveitamento dos alunos pode ser impactado por diversos fatores. Latgé, Araújo e Silva Junior (2020) ${ }^{(26)}$ e Silva et al. (2021) $)^{(5)}$ afirmam que além do caráter desafiador do ensino remoto, a qualidade do ensino também está relacionada a outros fatores, a saber: nível de conhecimento tecnológico, posse de recursos materiais tecnológicos, acesso à Internet e esforço dos sujeitos envolvidos, tornando-o inacessível para alguns, o que evidencia a fragilidade do ensino também atrelado à vulnerabilidade social.

De acordo com Hodges et al. $(2020)^{(23)}$, a alteração do ensino presencial para o on-line induz a um pensamento errôneo de que o aprendizado será fragilizado, entretanto, é a súbita transição dada as circunstâncias sanitárias que dificultou o proveito das possibilidades e utilização dos recursos. Ratificando isso, estudos indicam que as atividades a distância quando planejadas de forma adequada estimulam os alunos de forma igual ou até superior quando 
comparadas a aulas expositivas tradicionais, principalmente quando tecnologias interativas são $\operatorname{aplicadas}^{(5,25)}$.

Os achados deste estudo apontam que a realização de webinars, videoconferências, vídeos, lives e podcasts em programas como Zoom ${ }^{\circledR}$, Microsoft Teams ${ }^{\circledR}$, YouTube ${ }^{\circledR} e$ SoundCloud® foram proveitosas para cumprir com o componente acadêmico e que softwares como Kahoot! e Mentimeter ${ }^{\circledR}$ podem ser utilizados para aplicação de jogos e pesquisas, o que possibilita uma melhor interação entre os participantes de reuniões virtuais. Ademais, essas ferramentas permitem que atividades individuais ou em grupo sejam realizadas, potencializando a interação mesmo que de forma remota ${ }^{(18-19)}$.

Desse modo, esses resultados vão ao encontro dos achados de Damascena et al. $(2019)^{(27)}$, no que se refere à viabilidade do ensino por meio das tecnologias educacionais on-line, que atuam como agente facilitador do ensino remoto. Além de torná-lo prazeroso para os envolvidos, as ferramentas didáticas propiciaram a dinamicidade do processo de ensino-aprendizagem do ensino remoto, promoveram interatividade e contextualização da realidade e potencializaram o interesse e a autonomia dos alunos.

O melhor aproveitamento do ensino remoto relaciona-se com o método adotado. Segundo Sousa et al. $(2018)^{(28)}$, métodos de ensino que estimulam a participação ativa resultam em maior motivação e envolvimento dos discentes. Em concordância com isso, Konrad, Fitzgerald e Deckers $(2021)^{(21)}$ evidenciaram que a realização de discussões de casos clínicos por vídeo e demonstração de procedimentos por meio da simulação realísticas virtuais somadas a treino de habilidades de ausculta a partir da aplicação da tecnologia MedEdu À Easy Auscultation foi uma estratégia que oportunizou o desenvolvimento do raciocínio clínico dos acadêmicos, o que resultou em uma experiência exitosa.

Para a nova modalidade de ensino também foi necessário que técnicas de avaliação do desempenho acadêmico fossem adaptadas. O estudo de Elsalem et al. $(2021)^{(20)}$ identificou que os acadêmicos preferem a combinação de questionários e exames como método de avaliação. Quando questionados sobre o assunto, docentes que participaram de um estudo que envolveu professores brasileiros de diferentes níveis do sistema educativo afirmam que os métodos de avaliação mais utilizados são trabalhos de casa, avaliações com múltipla escolha com e sem consulta, avaliação dissertativa com consulta, avaliação formativa e observação do desempenho através de jogos ou simuladores on-line. Entretanto, os docentes declararam que não estão avaliando adequadamente seus alunos $^{(29)}$. Reforça-se, assim, a importância 
da avaliação formativa e não apenas as avaliações diagnósticas e somativa.

Em suma, este estudo reuniu e explorou os resultados obtidos por pesquisadores por meio da implementação de tecnologias educacionais no ensino remoto durante a pandemia de COVID-19, alcançando, assim, o seu objetivo proposto. Identificou-se que, mesmo com a necessidade de aperfeiçoamento dessa modalidade de ensino emergencial e temporária, a utilização de tecnologias educacionais digitais durante a pandemia possibilitou que os discentes continuassem sua formação acadêmica de forma remota. Ainda, permitiu a interação com os docentes e potencializou o processo de ensino-aprendizagem, respeitando as orientações de distanciamento social para evitar a aglomeração de pessoas que facilita a propagação do Sars-CoV-2.

\section{CONCLUSÕES}

Conclui-se que as tecnologias educacionais mais utilizadas foram encontros virtuais, podcasts, gamificação individuais ou em grupo, exames eletrônicos remotos, simulações realísticas virtuais e casos clínicos. Estas tecnologias quando utilizadas adequadamente tornam-se uma alternativa eficaz para a continuidade da formação acadêmica do enfermeiro durante a pandemia de COVID-19.
Ressalta-se que, quando empregadas no ensino da Enfermagem, as tecnologias precisam promover a discussão entre os envolvidos. Este método propicia o aprimoramento do raciocínio clínico, o compartilhamento de experiências e prioriza o protagonismo do discente. Entretanto, apesar das possibilidades proporcionadas pelas tecnologias digitais, a experiência prática nos serviços de saúde é indispensável para os discentes em formação.

Como principal limitação encontrada nesta revisão, aponta-se a escassez de estudos que respondessem à questão norteadora da pesquisa, levantando a hipótese de que algumas tecnologias utilizadas podem não terem sido publicadas. Esse problema pode ser compreendido em razão dos esforços dos pesquisadores estarem direcionados à resolução da atual crise sanitária. Entretanto, faz-se necessário que estudos sejam desenvolvidos com foco na qualidade do ensino, principalmente dos cursos de saúde. Além de melhorar a compreensão das experiências e conhecer a realidade de outros países.

No período de pandemia, o processo de ensino-aprendizagem na área da Enfermagem precisou ser ressignificado e reinventado. Assim, o presente estudo apresentou as potencialidades das tecnologias educacionais utilizadas durante o ensino remoto, contribuindo com a disseminação e 
discussão do conhecimento dessas tecnologias como uma possibilidade de ampliação da qualidade do ensino, do desenvolvimento do raciocínio diagnóstico e do estímulo ao diálogo e a criatividade dos discentes e docentes de enfermagem nesse contexto emergencial.

\section{REFERÊNCIAS}

1. Oliveira SS, Postal EA, Afonso DH. As Escolas Médicas e os desafios da formação médica diante da epidemia brasileira da COVID-19: das (in)certezas acadêmicas ao compromisso social. APS Revista. [Internet]. 2020 [cited 10 maio 2021]; 2(1):56-60. doi:https://doi.org/10.14295/aps.v2i1.69.

2. Ministério da Educação (Brasil). Portaria $\mathrm{n}^{\circ}$ 345, de 19 de Março de 2020. Autoriza, em caráter excepcional, a substituição das disciplinas presenciais, em andamento, por aulas que utilizem meios e tecnologias de informação e comunicação, por instituição de educação superior integrante do sistema federal de ensino. Diário oficial da União 19 mar. 2020.

3. Bertoldo HL, Mill D, Salto F. Dicionário crítico de educação e tecnologias e de educação a distância. Campinas: Papirus, 2018.

4. World Health Organization (WHO). [Internet]. Geneva: WHO: c2020 [cited 19 jun 2021]. Nursing and midwifery. January. Available from: https://www.who.int/newsroom/fact-sheets/detail/nursing-andmidwifery

5. Silva CM, Toriyama ATM, Claro HG, Borghi CA, Castro TR, Salvador PICA. Pandemia da COVID-19, ensino emergencial à distância e Nursing Now: desafios à formação em enfermagem. Rev Gaúcha Enferm. [Internet]. 2021 [cited 10 maio
2021]; 42(esp):e20200248.

doi: https://doi.org/10.1590/1983-

1447.2021.20200248

6. Corrêia DM, Morari DC, Borges JR. Do presencial ao não presencial. In:CASTRO, L. P. S. Cartilha do docente para atividades pedagógicas não presenciais. Disponível em:https://sead.paginas.ufsc.br/files/2020/04/ Cartilha-doDocente-APNP-UFSC.pdf. Acesso em: 10 maio 2021.

7. Brito G. da. S; PURIFICAÇÃO, I. Educação e novas tecnologias: um repensar. 2. ed. Curitiba: Intersaberes, 2015

8. Moreira JAM, Henriques S, Barros D. Transitando de um ensino remoto emergencial para uma educação digital em rede, em tempos de pandemia. Dialogia [Internet]. 2020 [cited 10 maio 2021]; (34):351-364. doi: https://doi.org/10.5585/Dialogia.N34.17123.

9. Oliveira ES, Freitas TC, Sousa MR, Mendes NCSGM, Almeida TR, et al. A educação a distância (EaD) e os novos caminhos da educação após a pandemia ocasionada pela Covid-19. Braz. J. of Develop [Internet]. 2020 [cited 10 maio 2021]; 6 (7):52860-52867. doi: https://doi.org/10.34117/bjdv6n7-799

10. Lira ALBC, Adamy EK, Teixeira E, Silva FV. Nursing education: challenges and perspectives in times of the COVID-19 pandemic. Rev Bras Enferm. [Internet]. 2020 [cited 10 maio 2021]; 73:e20200683. doi:https://doi.org/10.1590/0034-7167-20200683

11. Ministério da Saúde (BR). Conselho Nacional de Saúde. Resolução n. 573, de 31 de janeiro de 2018 [Internet]. 2018 [cited 08 Jun 2021]. Available from: http://conselho.saude.gov.br/resolucoes/2018/ Reso573.pdf

12. Pluye $\mathrm{P}$, Hong QN. Combining the power of stories and the power of numbers: mixed 
methods research and mixed studies reviews. Annu Rev Public Health [Internet]. 2014 [cited 10 maio 2021] 35(1):29-45. doi.org/10.1146/annurevpublhealth-032013182440

13. Cardoso V, Trevisan I, Cicolella DA, Waterkemper R. SYSTEMATIC REVIEW OF MIXED METHODS: METHOD OF RESEARCH FOR THE INCORPORATION OF EVIDENCE IN NURSING. Texto contexto-enferm. [Intenert]. 2019 [cited 17 Jun 2021]; 28, e20170279. doi: https://doi.org/10.1590/1980-265X-TCE2017-0279

14. Joanna Briggs Institute. Reviewers' Manual-Methodology for JBI Mixed Methods Systematic Reviews. [Internet]. Adelaide: JBI [cited 02 Abr 2021]. Available from: http://joannabriggs.org/assets/docs/sumari/Re viewersManual_Mixed-Methods-ReviewMethods-2014-ch1.pdf

15. Johnson N, Phillips M. Rayyan for systematic reviews. Journal of Electronic Resources Librarianship. [Internet]. 2018 [cited 02 Abr 2021] 30:1, 46-48. doi: 10.1080 / 1941126X.2018.1444339

16. Page M J, McKenzie J E, Bossuyt P M, Boutron I, Hoffmann T C, Mulrow C D, et al. The PRISMA 2020 statement: an updated guideline for reporting systematic reviews. The BMJ. [Internet]. 2021; 372 (71). doi:10.1136/bmj.n71

17. Melnyk BM, Fineout-Overholt E. Environmental health in public health community practice: An integrative review of the literature. Melnyk BM, Fineout-Overholt E, editors. Philadelphia: Lippincott Williams and Wilkins; 2005.

18. Silveira A, Santos NO, Wilhelm LA, Soccol KLS, Tisott ZL, Prates LA. Estratégias e desafios do ensino remoto na Enfermagem. Enfermagem em Foco. [Internet]. 2021; 11
(5). doi:https://doi.org/10.21675/2357707X.2020.v11.n5.4302.

19. Leigh J, Vasilica C, Dron R,Gawthorpe D, Burns E, Kennedy S, Kennedy R, Warburton $\mathrm{T}$, Croughan C. Redefining undergraduate nurse teaching during the coronavirus pandemic: use of digital technologies. British journal of nursing. [Internet]. 2020; 29 (10). doi:

https://doi.org/10.12968/bjon.2020.29.10.566.

20. Elsalema L, Al-Azzamb N, Jum'ahc AA, Obeidatd N. Remote E-exams during Covid19 pandemic: A cross-sectional study of students' preferences and academic dishonesty in faculties of medical sciences. Annals of Medicine and Surgery. [Internet] 2021; $62 . \quad$ doi: https://doi.org/10.1016/j.amsu.2021.01.054.

21. Konrad S, Fitzgerald A, Deckers C. Nursing fundamentals - supporting clinical competency on-line during the COVID-19 pandemic. Teaching and learning in nursing: official journal of the National Organization for Assciate Degree Nursing. [Internet]. 2020;16 (1). doi: https://doi.org/10.1016/j.teln.2020.07.005.

22. Mello JP, Vitorino AJR. Ensaio Crítico sobre as Possíveis Estratégias Adotadas para o Enfrentamento ao Fechamento das Escolas Provocado Pela Covid-19: rede municipal de ensino de Campinas (SP)-Brasil. Currículo sem Fronteiras. [Internet]. 2020; 20 (3).

23. Hodges C, Moore S, Lockee B, Trust T, Bond A. The difference between emergency remote teaching and on-line learning. Educause review. [Internet]. 2020; (27):1-12.

24. Lima EB, Paiva SC, Goulart JC. Ensino a distância frente à pandemia COVID-19. Revista de Estudos em Educação. [Internet]. 2021;1 (7): 20-31.

25. Silva ACO, Shirliane AS, Menezes JBF. $\mathrm{O}$ ensino remoto na percepção discente: 
desafios e benefícios. Dialogia. [Internet] 2020; (36):298-315. doi: https://doi.org/10.5585/dialogia.n36.18383

26. Latgé, PK, Araújo, DN, Silva Júnior, AG. Comunicação, educação e vigilância popular em saúde em tempos de COVID-19- a experiência das comunidades de Niterói, RJ. APS em Revista. [Internet]. 2020;2 (2): 122-127, 2020. doi:10.14295/aps.v2i2.110

27. Damascena SCC, dos Santos KCB, Lopes GSG, Gontijo PVC, Paiva MVS, Lima MES, Campos RS. Uso de tecnologias educacionais digitais como ferramenta didática no processo de ensino-aprendizagem em enfermagem. Brazilian Journal of Development. [Internet]. 2019;5 (12): 29925-29939. doi: https://doi.org/10.34117/bjdv5n12-13

28. Sousa NP, Lima JS, Epaminondas JM, Nunes EDCS. Ensino baseado em simulação: experiência no ensino de primeiros socorros em curso técnico. Revista Eixo. [Internet]. 2018; 7(2): 79-86.

29. Herrera VAS, Da Silva S, Dos Santos CER, Oliveira LP. Desafios docentes no contexto da Pandemia de COVID19: ferramentas e estratégias. Revista de Estudos e Pesquisas sobre Ensino Tecnológico (EDUCITEC). [Internet] 2020;6: e156420. doi:https://doi.org/10.31417/educitec.v6.1564.

\section{Autor correspondente}

Nome: Raphaella Castro Jansen;

Endereço: Rua José Franco de Oliveira, Centro, 62790000 - Redenção, CE - Brasil.

Telefone: (85) 98706-8628;

E-mail: raphaella.jansen@gmail.com.

Submissão: 2021-09-15

Aprovado: 2021-10-14 